\title{
INDEX
}

abstention from warfare $1-15,18,36$, 44

acquis communautaire 153, 166, 193

active labour market policy 92-6, 129

Adler, E. 42-7 passim, 54-6, 169

Ahern, Bertie 177, 194-5

Al Qaeda 196

Åland Islands 51, 70

alcohol consumption and taxation 175,200

Amsterdam Treaty (1997) 167-8, 193, 207

anarchy in international relations 37-41, 44-5, 48-51, 55, 73, 77, 90

Anderson, Benedict 169

Andrén, N. 5, 7, 72, 155, 182

Anschluß, 27, 71

armed neutrality 15-17, 28, 37-9, $50,54,61,72,105$

arms control 28, 55, 156

Åström, Sverker 82-3, 101, 106, 115 , $134,151,158,181,191$

Athenian politics 12, 37

Austria 6, 25, 27, 41, 49-50, 100, 151-3, 176-7, 192-5, 198

balance of power, international 16 , $21-7,38,40,50,52,101-2$, 107, 206

within Sweden 64

Barkin, J.S. 34, 40

Bartelson, J. 40, 55

Belgium 17, 38, 41, 105, 190

Berger, P.L. 43, 47-8, 99, 160

Bergman, Ingmar 83

Bernadotte, F. 80, 108-9

Bildt, Carl 109, 121, 128, 138, 142-3, 147-61 passim, 165-7,
172-3, 180, 183, 199-200, 207

Bin Laden, Osama 196

Binter, B. 32, 35

Blair, Tony 46, 179, 195, 197

third way $174-5$

Blyth, M. 123, 127-8, 132, 159, 161

Bohlin, Britt 151, 159, 181

Branting, Hjalmar 67, 69-70

Britain 41, 45-7, 56, 69, 84, 125, $131,135,171,179$

Bukovansky, M. 52, 105

Bush, George W. 2, 189-203 passim, 206

business interests 128-9, 132, 134, $138,149,152,159,174,207$

Carlsson, Ingvar 35, 112, 130, 146, $148,165,173,191-2$

Carr, E.H. 18-19, 21

censorship 80, 84

Childs, Marquis 73-4

Christianity 12-13, 29

Churchill, Winston 19, 22, 69, 79, 81

Clinton, Bill 202

co-determination 123, 126

'cognitive evolution' 43, 54

'cognitive frames' 159

'cognitive regions' 169

Cold War 25, 28-9, 90, 102, 104, $107-11,120$

ending of 1, 32-5, 50, 56, 143146 , 206

collective security 19-20, 25, 54, 71

Commission on Neutrality Policy 151

Common Foreign and Security Policy (CFSP) 7, 36, 142-5, 152-4, 166-71, 176, 192, 197, 207

Conference on Security and Cooperation in Europe (CSCE) 
$28,106,111,154$

conflict resolution 35, 51, 92, 110 , $115,128,203$

consensualism 75, 92, 97, 121, 137, 203

constructivism see social constructivism

Cook, Robin 179

Council of Europe 154, 167

Cox, M. 24, 35, 153

Cronin, B. 34, 40

cruise missiles 28,111

culture, definition and production of 43

Czech Republic 170-1

Czechoslovakia 102

Dagens Nyheter 128, 172

Davignon Report 135-6

December Compromise (1906) 67-8

decommodification 95, 98, 175

Delanty, G. 100, 170-1

Delors, Jacques 136

democratic deficit 170

Denmark 15, 22, 26, 40, 63, 102-4, $107,124,132,175,179-80$, 195

Deutsch, K. 55, 107, 170

devaluation 130

direct democracy 53

disarmament 70, 110-12, 115-16

Dulles, John Foster 25

Dunne, T. 146, 190, 202-3

Dürrenmatt, Friedrich 4

Economic and Monetary Union (EMU) $40,174,200$

Eisenhower, Dwight D. 25

epistemic communities 55-6

Erlander, Tage 92, 94-5, 109, 113, 135

Esping-Andersen, G. 95, 132, 137

Estonia 175

Ethiopia 19, 71

ethnic conflicts 33, 189

Eurobarometer surveys 174, 202
European arrest warrant 196-7

European Free Trade Association

(EFTA) 106, 136, 145, 153

European identity 169-71,

174-6, 181

European Rapid Reaction Force

168

European Trade Union Confederation 125

European Union (EU) 2, 7, 27,

$33,36,40,45-7,55-6,106$,

134-6, 139, 142-60, 165-81,

188-203, 207

proposed constitution

93-4, 198, 200

Fälldin, Thorbjorn 124, 129, 135, 158

film industry 83

Finland 6, 26, 28, 41, 49, 51, 62, 69, 76-80, 100-4 passim, 151-4, $160,167-8,171,175-6,179$, $182,194-5,198$

Finnemore, M. 43, 159

First World War 17, 20, 28-9, 68-9

FitzGerald, Garret 177

folkhem (People's Home) 7, 47-8, 60, 65, 73-6, 82-3, 94-5, 98-9, 106, 113-14, 120-2, 129,

$139,147,155,207$

France 41, 125, 198-201

full employment 93, 145

Furubjelke, Viola 151, 158

de Gaulle, Charles 135

Germany 17, 19, 27, 69-72, 78-81, $84-5,125$

globalisation 7, 33, 41, 125, 137-9, 157-8, 172, 179-80, 188-9, $198-201,208$

Gothic Society 63

Greece, Ancient 11-12

Grotius, Hugo 14-15, 18, 22

Group of 21156

Gustav V, King 69, 81

Gustavus Adolphus, King 10, 54 
Hägglöf, Gunnar 23, 82, 101-2

Hague Conventions 16, 19, 24, 29, 78-9, 84, 155

Hakovirta, H. 29-30, 49-50

Hall, Anders 147, 154

Hammarskjöld, Dag 108-9, 115

Hampden-Turner, C., 73-4, 99-100

Hansson, Albin 73-6, 82, 92, 97-8

'harmony of interests' doctrine 18-19

Heclo, H. 67, 73-5, 91, 93

Hedin, N. 80, 82

Herodotus 12

Himmler, Heinrich 80

'historical continuity' of Swedish neutrality 60

Hitler, Adolf 23, 72, 82

Hjelm-Wallén, L. 173, 180, 199

Home Guard 71, 82

Huldt, B. 35, 106, 154, 192

human rights 115,144 , 196-7

humanitarian interventions 33,

112-13, 167-8, 189, 202

Hungary 144

Huntington, S. 34, 189

Hussein, Saddam 196

Iceland 104, 107

idealism in international

relations 3-4, 17-18, 21, 39

impartiality between bellligerents 16 , 23

India 108

individualism 199-200

industrial policy 123

insularity 61-2

'integral neutrality' 79

International Criminal Court 196

international institutions 33, 54, 105

international law 14, 16, 20, 24, 29, $39,41,72,107-10,115,155$, 189, 196-7, 202

International Monetary Fund 103

international relations (IR)

theory 3-4, 10, 20-1, 24, 30, 34, $41-2,55-7,208$

internationalism 6, 19-20, 53, 70, 72,
$85,91,101,107-10,114-15$,

$120,155-8,195-8,202-3$

radical 113

Iran 109

Iraq 109, 196-8, 202

Ireland 22, 45, 50, 152, 76-7, 194-5

isolationism 20, 32, 41, 52, 61-2, 73,

$81,85,103,120,190,206-8$

Israeli-Palestinian conflict 109, 190

Italy 19, 46, 71

Jackson, R. 37, 40

Jakobson, Max 49, 176

Japan 19

Jefferson, Thomas 52

Jenson, J. 122, 138

Joenniemi, P. 5, 36-8, 50-1, 107, 179-80

Joesten, J. 77-8, 81, 84

'just war' doctrine 14-15, 18-19, 29, 190

Kant, Immanuel 18, 170

Karl VII, King 64

Karl Johan, King 60-3, 85, 108-9

Karlsson, B. 84, 103

Karsh, E. 16, 19, 23, 45

Katzenstein, P.J. 43, 52

Kellogg-Briand Pact 53

Keynesian economics 92-3, 124-5, 130-1

Khrushchev, Nikita 26-7

Klestil, Thomas 153, 176-7

Korean War 107-8

Korpi, W. 77, 92, 132, 137

Kreisky, Bruno 49-50, 74

lagom idea 100, 122

Landsorganisationen Sverige (LO) 67-8, $77,121-3,126,130-3,138$, 145,175

language 47

Latvia 175

Lawler, P. 76, 98, 160

League of Nations 17-21, 24, 51, 70-2, 101 
Leagues of Armed Neutrality 15-16, 61

Lebanon 109

Lindbeck, A. 93, 132, 137

Lindbeck Commission 122, 148-9

Lindh, Anna 171, 173, 179, 181, 191-2, 196-7, 200

Lloyd George, David 69

Logue, J. 70, 74, 81

Luckmann, T. 43, 47-8, 99, 160

Luxembourg 22

Maastricht Treaty 40, 144, 152, 167

McCracken Report 125

MacDougall Report 125

MacGinty, R. 24, 35, 153

Machiavelli, Niccolò 13, 21

Madsen, H. 67, 73-5, 91

Mahon, R. 122, 138

Malmborg, af M. 62-3

Malta 178, 195

Marcussen, M. 149, 160-1

Marshall Plan 102

Marxism 67

means-testing 95

mediation 110, 116, 157, 167, 182-3

Meidner, Rudolph 73, 92-6, 121, 130, 159

Melian Dialogue 11-12, 17, 22, 37

metaphors 47-8, 98, 129

Mintoff, Dom 178, 195

monetarism 132

Morgenthau, H.J. 17, 20-3, 26

Mouritzen, H. 49, 65, 180

Myrdal, Alva 92, 98-9

Myrdal, Gunnar 74, 92, 98-9, 113

myths 47-8, 90, 160, 182, 207

national identity 5, 45, 57, 101, 156

Swedish 7, 63, 85, 166, 184, 207

national interests 44, 152, 167

nationalism 134, 139

nation-states, building of 51, 61

Nazism 84

neoclassical economics 132, 138 neoliberalism 114, 124-8,

132-3, 136-9, 158, 161,

165, 199-200, 207

neo-realism 27-8, 34, 52, 54

Netherlands, the 22

Neuhold, H. 27, 49

Neumann, Iver B. 47, 134

neutralism as distinct from neutrality 25

neutrality, active form of 105, 108-9

challenges to $1-2,41$

common perceptions of $1,4,10$

consensus of $110,150,155$

definition of 50

diminishing use of phrase 151

of European countries

176-8, 193-6

as an issue of identity 48

literature on $2-5,12,72-3,82-3$

obsolescence of 183, 188, 208

positive and negative 3

problem with the concept of 2, 37-8

public support for 105

status of 22, 35-9

Swedish variety of $6-8,18,35,90$, 101, 116, 201, 207

symbolism of 183

new international economic order 114

New Zealand 132

Nixon, Richard 115-16

non-alignment policy $24,26,35,111$, 150-1, 169, 173, 176-8, 191-5

Nordic cooperation 106-7,

$134,178-83,195$

norms 42-4, 48, 52-4, 107, 110-11,

115-16, 139, 161, 165, 175,

181-4, 196-203 passim, 206-9

North Atlantic Treaty

Organisation (NATO) 7, 28,

33-5, 102-4, 107-11, 135,

$143-3,154-6,160,166$,

171-3, 176-7, 182, 189-98

Northern Dimension project 179

Norway 17, 22, 26, 62, 68, $76,79,102-7,124,166$, $180,183,190,195-8$ 
nuclear technology 28, 102, 110

nuclear test bans 110-12

Oberg, J. 192, 201

oil crises 124, 130, 133

overseas development assistance 112-13, 116

\section{Pakistan 108}

Palm, August 66

Palme, Olof 82, 90, 92, 99, 101, 109-12, 115-16, 120, 122, 135,178

Palme Commission 112

Palme Doctrine 28

pan-Scandinavianism 63, 107

peace research 109

'peaceful coexistence' doctrine 26

peacekeeping operations 109-10,

116, 154, 167-9, 173, 178, 194

Peloponnesian War 11, 29, 41

Persson, Göran 171-5, 179, 181, 184, 188, 191-2, 196-200, 207

Pestoff, V.A. 125, 148

Petersberg tasks 167-9, 183, 193, 207

Poland 77

Portugal 22, 199

positivism 15-17

postmodernism 189

power relations, international 21 , 202-3

see also balance of power

Press Council 80

privatisation 134, 147-8

proclamations of Swedish neutrality $61-2,69,77$

Prodi, Romano 189

public sector employment 129

Pufendorf, Samuel 15, 18

Putin, Vladimir 190

quasi-neutralism 25

Rasmussen, Anders Fogh 180, 195

rationalism 50
Reagan, Ronald 28, 125, 130

realism in international relations $3-4$, 12, 17-18, 21-4, 28, 34-41, 44, 48-52 passim, 55, 71-3, 90, 112, 142,150

referenda 165-6, 194, 200-1

Reformation, the 14, 54

regions within nation-states 46

Rehn, Gösta 92-6, 121, 130, 159

Rice, Condeleeza 202

rights of neutrals 15-18, 22, 29, 37, 83

Riksdag, the 64, 66, 71, 75-7, 91, $101,104,112,122,136,146-7$. 150, 181-2, 190, 192, 196

Romanticism 63

Roosevelt, F.D. 20, 74

Roscher, R. 149, 160-1

Ross, J.F.L. 36, 135

rule of law 18, 70, 144

Russia 63, 69, 78, 84, 154

see also Soviet Union

Ruth, A. 63-5, 68, 71, 84, 100, 160, 180

Rydén, B. 131, 137

Ryner, M. 95, 127-8, 132, 149

Sainsbury, D. 92, 97

Salmon, P. 20, 39

Saltsjöbaden Agreement 77, 92, 123, 126

sanctions 19, 24

Scandinavian Defence Union, planned 102-4

Scandinavian Movement 63

Schengen Information System 197

Schori, P. 192, 196, 199

Schüssel, W. 176, 192

sclerosis thesis 132

Scotland 47

Second World War 21-4, 29, 77-85, 177

self-interest of neutral states 23, 120, 206

September 11, 2001 attacks 2, 188-91, 194, 201 
Sikkink, K. 43, 159

Single European Act 136

Skandia (company) 159

social constructivism $5-7,30,32-3$, $41-8,54,57,90,121,159,166$, $181,183,207$

thick and thin varieties of 42

Social Democracy 48, 66-70, 73, 82, 85, 90-2, 97, 100, 108, 112-16, 122-6, 130-9, 142, 145, 148-51, 154-61, 165-6, 172-5, $179,183,191,198-9,207$

definition of 74

Swedish variety of 74-5

see also Socialdemokratiska Arbetarepartiet

social stratification 95

Socialdemokratiska Arbetarepartiet (SAP) 6, 60-1, 66-9, 73-7, 90-101, 105-6, 114-15, 120-35, 138-9, 145-7, 150-1, 156-61, 165-6, 173-5, 180-1, 191-2, 200, 207

hegemony of $91,142,145,181$

Third Way Policy 121, 129-32 socialisation 45 socialism $66-7,73-5,93-4,112-13$ Södersten, B. 114, 157

'soft' security 144, 168-9, 202

Solana, Javier 188, 195, 197

solidarity, 6, 7, 35, 48, 60-1, 72, 76, 77, 82-3, 90, 92, 94, 98-9, 101, 104, 106-7, 108, 111, 113, 115. $116,121,183,198,199$

sovereignty 14-17, 27, 35-41, $44,81-4,104,106,135,145$, 188, 199, 203, 208

Soviet Union (USSR) 19, 25-7, 49, 56, 102-3, 111, 143, 151-2

see also Russia

Spain 22, 199-200

Spanish Civil War 71

Stalin, Joseph 82-3

Stern, M. 3, 70, 102, 116

Stockholm Conference (1938) 78

Stockholm Exhibition (1897) 98-9
Stråth, B. 60, 65, 125, 170-1

strike action 122-3, 131, 146

Strindberg, A. 64

Stromberg, P.G. 64-6

Sundelius, B. 28, 62, 70, 113, 150

Svenska Arbetsgivareföreningen (SAF) $67,123,127-9,133,148$

Svenska Dagbladet 128

Swedish Model 6, 66, 74, 90, 92-8, 100-1, 105, 120-2, 129, 131-8 passim, 145-9 passim, 161, 174. 198,200

Switzerland 4, 18-28 passim, 41, 44, 47, 53-4, 177-8, 193

Tegnér, Esaias 62, 76

terrorism and the 'war on terror' 2, 188-98, 201-2, 206, 208

Thatcher, Margaret 125, 130, 136, 149

Thirty Years War 54, 61

Thucydides 10-12

Tilton, T. 92, 94, 137

Touraine, A. 43, 199

trade, neutrality in 13-16, 61, 68-9, 78,84

trade unions 131, 199

Transito affair 69

Trompenaars, F. 73-4, 99-100

Truman, Harry 25

Trumam Doctrine 102

Undén, Östen 70, 82, 101-2, 105, 110, 115, 151

'Third Way' 105, 110

United Kingdom see Britain

United Nations 28, 54, 70, 90, 101-15, 154-8, 167, 171, 173, 178-9, 191-3, 197, 202

Charter 24, 108, 198

Conference on Trade and Development 114

General Assembly 25, 107-8, 115, 156

Security Council 24, 101, 103, 107-8 
Index

United States 17, 19, 25-8,

$46,52,56,69,74,83,103-4$, $115,125,196-8,202-3$

National Security Council 25

War of Independence 16

universalism, 6, 7, 20, 66, 76, 91, 92, 94-6, 97, 98, 99, 111, 112-14, 116,175

Vattel, Emmerich de 15, 18

Väyrynen, R. 24, 39-40, 51

Versailles Treaty 52

Vienna, Congress of 16

Vietnam War 83, 115-16

wage earner funds 123-30

wages policy 96,130

Wahlbäck, K. 62, 157

Wallace, W. 45-6, 57
Waltz, K.N. 21, 27-9, 54, 143

war, causes of 21, 110

Warsaw Pact 33, 102

Washington Treaty 189

Weiss, L. 121-2, 137

welfare state $47,66,75,94-5,98$, 100, 113-14, 121-2, 129, 131-8 passim, 145-9 passim, 161, 174, 198,200

Wendt, A. 42-4, 48-51, 63, 85, 156

Werner Report 135-6

Western European Union (WEU) 144, 153-4, 166-71, 177

Westphalian state system 10, 15

Wigforss, Ernst 76, 92, 96, 123

Wilson, Woodrow 17-18, 22, 52, 69

Wolff, Christian 15, 36

Zetterberg, H.L. 128-9 